\title{
Floristic composition and phytogeography of the tree component of Araucaria Forest fragments in southern Brazil
}

\author{
PEDRO HIGUCHI ${ }^{1,2}$, ANA CAROLINA DA SILVA ${ }^{1}$, TIAGO SOUSA FERREIRA ${ }^{1}$, \\ SHEILA TRIERVEILER DE SOUZA ${ }^{1}$, JULIANO PEREIRA GOMES ${ }^{1}$, \\ KARINA MONTIBELLER DA SILVA ${ }^{1}$ and KRISTIANA FIORENTIN DO SANTOS ${ }^{1}$
}

(received: March 27, 2011; accepted: April 17, 2012)

\begin{abstract}
Floristic composition and phytogeography of the tree component of Araucaria Forest fragments in southern Brazil). The present study examined the floristic composition of three fragments of Araucaria Forest (AF) in the Planalto Catarinense region of southern Brazil as well as the floristic contextualization of these areas in relation to other remnant AF sites. Three AF fragments at different altitudes were analyzed in the municipalities of Campos Novos, Lages, and Painel. Fifty $200 \mathrm{~m}^{2}$ plots were examined in each fragment and all of the trees with $\mathrm{CBH}$ (circumference at breast height) $\geq 15.7 \mathrm{~cm}$ were identified. In order to floristically contextualize the study fragments, comparisons were made with other remnant AF sites by way of dendrograms and NMDS (Non-metric multidimensional scaling). Environmental and spatial variables were plotted on the diagram produced by the NMDS to evaluate their influence on the floristic patterns encountered. The forest fragments studied demonstrated high floristic heterogeneity, indicating that AFs cannot be considered homogeneous formations and they could be classified into 3 phytogeographical categories: i) high altitude areas influenced by cloud cover/fog, including the Painel region; ii) areas of lesser altitude and greater mean annual temperatures situated in the Paraná River basin, and iii) areas situated in the Paraná and Upper-Uruguay river basins and the smaller basins draining directly into the southern Atlantic, near Campos Novos and Lages. The environmental variables most highly correlated with species substitutions among the sites were altitude, mean annual temperature, and the mean temperature of the most humid trimester.
\end{abstract}

Key words - Araucaria Forest, arboreal species, environmental variables

\section{INTRODUCTION}

Mixed Ombrophilous Forests (MOF) (IBGE 1992), also known as Araucaria Forests (AF), represent one of the principal plant formations in southern Brazil. These forests predominate on the Brazilian Meridional Plateau, principally on the southern Catarinense Plateau, between $24^{\circ}$ and $30^{\circ} \mathrm{S}$ latitude at altitudes that vary between 500 and $1400 \mathrm{~m}$ above sea level, with disjunct occupation of sites in elevated areas of the Serra do Mar and Mantiqueira Mountains in southeastern Brazil (Klein 1960). Their largest extensions cover the hydrographic basins of the rivers that drain into the Uruguay and Paraná rivers, following the altitudinal gradients from the higher lands of the Serra do Mar and Geral Mountains towards the interior (west). AFs, like most other Brazilian forests, are currently highly fragmented due to historical anthropogenic use and occupation of the land for agriculture, cattle raising, and (more recently) large-scale monoculture

\footnotetext{
1. Universidade do Estado de Santa Catarina (UDESC), Centro de Ciências Agroveterinárias, Departamento de Engenharia Florestal, Av. Luiz de Camões, 2090, Bairro Conta Dinheiro, 88520-000 Lages, SC, Brazil.

2._Corresponding author: higuchip@gmail.com
}

plantations of Pinus spp. These activities have reduced these once extensive and continuous forests to isolated fragments with sizes and levels of disturbance that vary from one locality to another (Klein 1978, Behling \& Pillar 2007).

Araucaria angustifolia is the most characteristic species of the AF phytophysiognomy as it forms the upper canopy and has a dominant character in this vegetation. In spite of the fact that $A$. angustifolia predominates in the upper forest canopy (Leite \& Klein 1990), the middle and lower strata demonstrate large diversities of species such as Ocotea porosa (Nees \& Mart.) Barroso (imbuia), Luehea divaricata Mart. (açoita-cavalo), Cedrela fissilis Vell. (cedro), Dicksonia sellowiana (xaxim), and Maytenus ilicifolia Mart. ex Reissek (espinheira-santa) (Nascimento et al. 2001, Behling \& Pillar 2007).

In light of the significant environmental heterogeneity in this region, associated principally with the climate, edaphic factors, and the history of anthropogenic disturbances, it would be expected that different AF fragments would show large floristic variations. According to Klein (1978), Santa Catarina State can be divided into four distinct phytophysiognomic areas: i) Araucaria Forests in the Iguaçu-Negro basin and in the upper regions of the river basins that drain into the 
Uruguay River; ii) Araucaria Forests of the PelotasCanoas basin; iii) Araucaria Forests of the extreme west; and iv) Araucaria Forest Nuclei of the Atlantic Rainforest. According to the classification system suggested by IBGE (1992), this phytophysiognomy may be subdivided according to its altitude and its proximity to water courses into Alluvial, Sub-Montane, Montane, and High-Montane formations.

The Catarinense Plateau is considered a strategic region for forest conservation as it contains numerous headwaters that form the hydrographic basin of the Uruguay River (Sgrott 2003) and is also part of the recharging area of the Guarani aquifer (Gomes et al. 2006). Little is currently known about the floristic and phytogeographical patterns of the arboreal components of AF forest fragments (Klein 1978, Formento et al. 2004) or their interactions with environmental and spatial variables. As such, the present study was designed to: i) describe the floristic composition of three AF fragments in the Catarinense Plateau region of southern Brazil; ii) determine the floristic similarities between these fragments in relation to other AF areas in southern Brazil; and iii) evaluate the influence of environmental and spatial variables on the phytogeographical patterns encountered.

\section{MATERIAL AND METHODS}

\section{Study areas}

Three AF fragments located at different altitudes in the southern Catarinense Plateau were investigated. These fragments were located in the municipalities of Campos Novos $\left(27^{\circ} 35^{\prime} 09.69^{\prime \prime} \mathrm{S}\right.$ and $51^{\circ} 09^{\prime} 15.07^{\prime \prime} \mathrm{W}$, altitude $712 \mathrm{~m}$, area $=1,551.37 \mathrm{ha})$, Lages $\left(27^{\circ} 51^{\prime} 19.20^{\prime \prime} \mathrm{S}\right.$ and $50^{\circ} 10^{\prime} 33.39^{\prime \prime} \mathrm{W}$, altitude $993 \mathrm{~m}$, area $=103.06 \mathrm{ha}$ ), and Painel $\left(27^{\circ} 55^{\prime} 27.56^{\prime \prime} \mathrm{S}\right.$ and $49^{\circ} 56^{\prime} 30.14^{\prime} \mathrm{W}$, altitude $1,196 \mathrm{~m}$, area $=48.91 \mathrm{ha}$ ) (figure 1). According to the WORDCLIM databank (Hijmans et al. 2005), the mean annual precipitation levels in these fragment areas (from 1960-1990) were 1719, 1483, and $1569 \mathrm{~mm}$ in Campos Novos, Lages, and Painel respectively, with rainfall being well-distributed throughout the year. The mean annual temperatures (1960-1990) in these fragments were 17.1, 16.1 , and $15{ }^{\circ} \mathrm{C}$ respectively. The climates in all three study areas were classified as $\mathrm{Cfb}$, following the classification system of Köppen, being characterized by rainfall uniformly distributed throughout the year and without a dry season. The fragments studied were areas within the Canoas and Pelotas River hydrological basins, and their topography is, for the most part, slightly undulating to undulating. According to the vegetation classification system of IBGE (1992), the arboreal vegetation of the Catarinense Plateau region can be classified

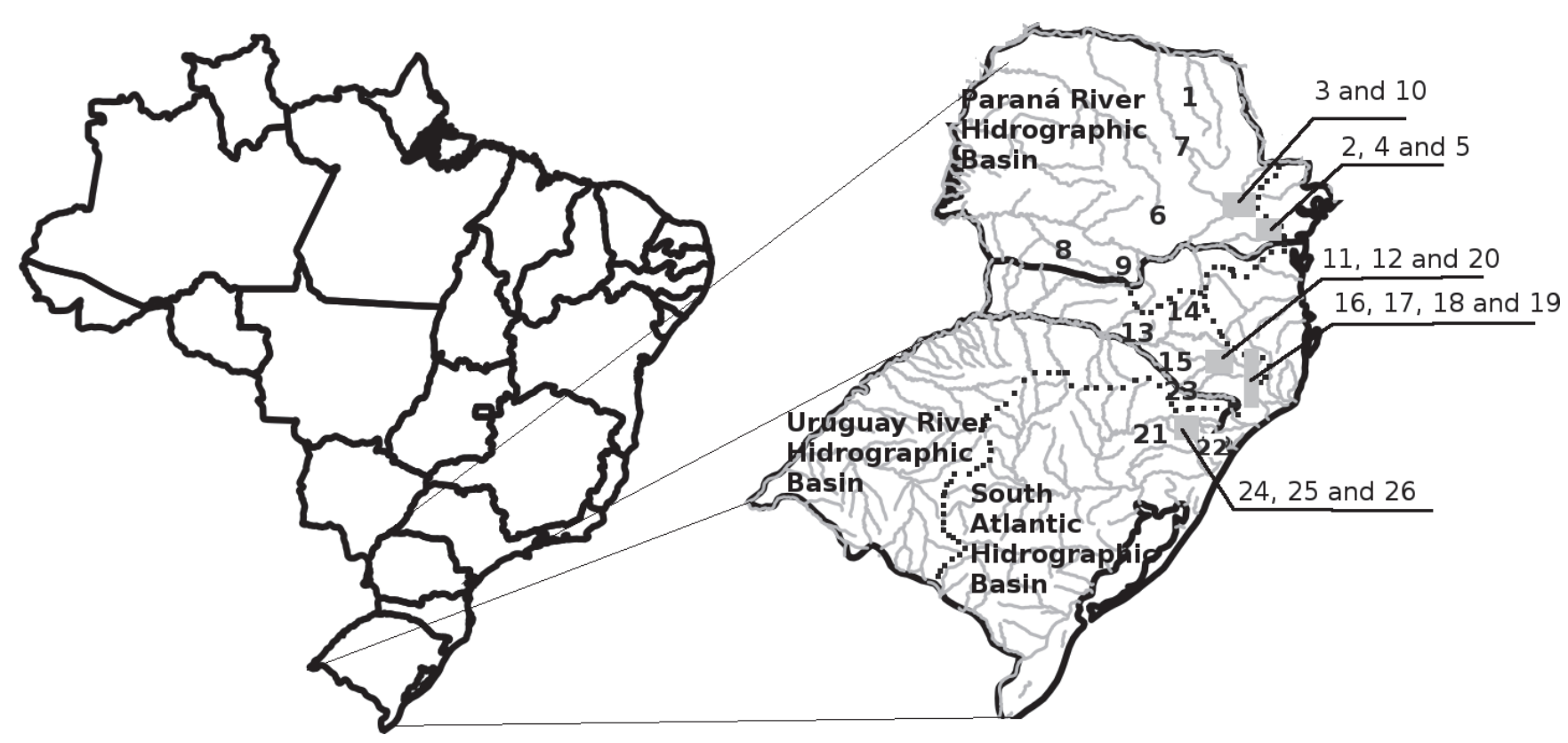

Figure 1. Location of the Araucaria Forest fragments in southern Brazil. 1. Arapoti, PR; 2. Pinhais, PR; 3. São João do Triunfo, PR; 4. Curitiba, PR; 5. Curitiba, PR; 6. Irati, PR; 7. Tibagi, PR; 8. Clevelândia, PR; 9. General Carneiro, PR; 10. Palmeira, PR; 11. Lages, SC; 12. Painel, SC; 13. Campos Novos, SC; 14. Caçador, SC; 15. Campo Belo do Sul, SC; 16. Bom Jardim da Serra, SC; 17. Urubici, SC; 18. Urubici, SC; 19. Bom Jardim da Serra, SC; 20. Lages, SC; 21. Nova Prata, RS; 22. São Francisco de Paula, RS; 23. Bom Jesus, RS; 24. Caxias do Sul, RS; 25. Vacaria, RS; 26. Criúva, RS; 27. São José dos Ausentes, RS. 
as Montane and High-Montane Mixed Ombrophilous Forest. The fragments examined had different histories of use and disturbance, but like most of the AF remnants in the state, all of them had experienced intense harvesting of both lumber and non-lumber species during the 1960's.

The surveys of the floristic compositions of the arboreal components of the sites involved establishing 50 permanent plots $\left(10 \times 20 \mathrm{~m}=200 \mathrm{~m}^{2}\right.$ each $)$, totaling 1 ha, to sample each fragment area. The permanent plots were distributed (with $30 \mathrm{~m}$ spacing between them) along parallel transects through the forests. The spatial arrangements of these transects were designed to adequately sample the environmental variables present in the areas, such as edge $\times$ interior and other variations related to soil types and altitudinal and topographic gradients. As such, the numbers and lengths of the transects varied among the fragments as a function of the unique characteristics of each, taking into consideration their spatial parameters (e.g. shape and total area) and environmental heterogeneity (e.g., topography). All of the arboreal individuals in each plot with $\mathrm{CBH}$ (circumference at breast height, measured $1.30 \mathrm{~m}$ above soil level) $\geq 15.7 \mathrm{~cm}$ were identified and marked with aluminum tags. Plant identifications were made by comparing them with herbarium samples and through consultations with specialist and the use of specialized literature. The angiosperm species were classified into families and genera according to the APG III system (2009). The botanical material collected was incorporated into the Santa Catarina State University herbarium (LUSC).

\section{Phytogeographical contextualization}

In order to phytogeographically contextualize the study localities, previous scientific publications of floristic lists and surveys of arboreal species of remnant Araucaria forests in southern Brazil were consulted (table 1). The information concerning each species was subject to a detailed revision with respect to habitat, nomenclature, and taxonomic synonyms (which were verified on the W3 Tropicos site) (Mobot 2010). The analyses did not include plants identified only to the family or genus level.

To analyze the relationships between the floristic compositions of the areas in terms of their environmental

Table 1. Floristic and phytosociological surveys of Araucaria Forest fragments in southern Brazil using cluster and ordination analyses. * = Identification codes for the areas examined in the dendrogram and clustering analyses using NMDS analyses; s.m. = Sampling method; i.l. = Inclusion level; № esp. = number of species; $\mathrm{CBH}=$ circumference at breast height; $\mathrm{DBH}=$ diameter at breast height; $\mathrm{PCQ}=$ point centered quarter.

\begin{tabular}{|c|c|c|c|c|c|c|c|}
\hline$*$ & Locality & Municipality & Source & s.m. & i.l. & № sp. & Climate \\
\hline $\operatorname{arapPR}$ & Rio das Cinzas & Arapoti, PR & Blum et al. 2003 & Plots & $\mathrm{DBH} \geq 10 \mathrm{~cm}$ & 134 & Cfa \\
\hline pinPR & $\begin{array}{l}\text { Condomínio Residencial } \\
\text { Alphaville Graciosa }\end{array}$ & Pinhais, PR & Seger et al. 2005 & Plots & $\mathrm{CBH} \geq 15 \mathrm{~cm}$ & 44 & $\mathrm{Cfb}$ \\
\hline sjtPR & $\begin{array}{l}\text { Estação Experimental de } \\
\text { São João do Triunfo }\end{array}$ & $\begin{array}{l}\text { São João do } \\
\text { Triunfo, PR }\end{array}$ & $\begin{array}{l}\text { Sanquetta et al. } \\
2000\end{array}$ & Plots & $\mathrm{DBH} \geq 10 \mathrm{~cm}$ & 66 & $\mathrm{Cfb}$ \\
\hline cur1PR & Capão do Tigre & Curitiba, PR & $\begin{array}{l}\text { Rondon Neto et al. } \\
2002\end{array}$ & Plots & $\mathrm{DBH} \geq 5 \mathrm{~cm}$ & 77 & $\mathrm{Cfb}$ \\
\hline cur2PR & $\begin{array}{l}\text { Parque Municipal do } \\
\text { Barigüi }\end{array}$ & Curitiba, PR & Kozera et al. 2006 & PCQ & $\mathrm{CBH} \geq 10 \mathrm{~cm}$ & 97 & $\mathrm{Cfb}$ \\
\hline iraPR & $\begin{array}{l}\text { Parque Ambiental Rubens } \\
\text { Dalle Grave, }\end{array}$ & Irati, PR & $\begin{array}{l}\text { Valério et al. } \\
2008 b\end{array}$ & Plots & $\mathrm{DBH} \geq 10 \mathrm{~cm}$ & 39 & $\mathrm{Cfb}$ \\
\hline tibPR & Fazenda Batavo & Tibagi, PR & Dias et al. 1998 & Plots & $\mathrm{DBH} \geq 5 \mathrm{~cm}$ & 127 & Cfa \\
\hline clePR & Clevelândia & Clevelândia, PR & $\begin{array}{l}\text { Valério et al. } \\
2008 \mathrm{a}\end{array}$ & Plots & $\mathrm{DBH} \geq 20 \mathrm{~cm}$ & 26 & $\mathrm{Cfb}$ \\
\hline $\mathrm{gcPR}$ & $\begin{array}{l}\text { Faz. Indústrias Pedro N. } \\
\text { Pizzatto Ltda }\end{array}$ & $\begin{array}{l}\text { General Carneiro, } \\
\text { PR }\end{array}$ & $\begin{array}{l}\text { Watzlawick et al. } \\
2009\end{array}$ & Plots & $\mathrm{DBH} \geq 10 \mathrm{~cm}$ & 39 & $\mathrm{Cfb}$ \\
\hline palPR & Três Morros & Palmeira, PR & Iurk 2008 & Plots & All fertile phanerogams & 134 & $\mathrm{Cfb}$ \\
\hline lagpbSC & Pedras Brancas & Lages, SC & This study & Plots & $\mathrm{DBH} \geq 5 \mathrm{~cm}$ & 92 & $\mathrm{Cfb}$ \\
\hline paiSC & Farofa & Painel, SC & This study & Plots & $\mathrm{DBH} \geq 5 \mathrm{~cm}$ & 49 & $\mathrm{Cfb}$ \\
\hline cnovSC & Ibicuí & Campos Novos, SC & This study & Plots & $\mathrm{DBH} \geq 5 \mathrm{~cm}$ & 87 & $\mathrm{Cfb}$ \\
\hline cacSC & $\begin{array}{l}\text { Reserva Florestal } \\
\text { Embrapa-Epagri }\end{array}$ & Caçador, SC & $\begin{array}{l}\text { Negrelle \& Silva } \\
\text { 1992; Lingner et } \\
\text { al. } 2007\end{array}$ & $\begin{array}{l}\text { PCQ and } \\
\text { plots }\end{array}$ & $\begin{array}{c}\mathrm{DBH} \geq 5 \mathrm{~cm} \text { and } \mathrm{CBH} \geq \\
60 \mathrm{~cm},\end{array}$ & 62 & $\mathrm{Cfb}$ \\
\hline cbsuSC & Capatazia Picassos & $\begin{array}{l}\text { Campo Belo do Sul, } \\
\text { SC }\end{array}$ & $\begin{array}{l}\text { Formento et al. } \\
2004\end{array}$ & Plots & $\mathrm{DBH} \geq 10 \mathrm{~cm}$ & 60 & $\mathrm{Cfb}$ \\
\hline
\end{tabular}




\begin{tabular}{|c|c|c|c|c|c|c|c|}
\hline * & Locality & Municipality & Source & s.m. & i.l. & № sp. & Climate \\
\hline bjseSC & $\begin{array}{l}\text { Estancias Ribeiro and } \\
\text { Machado }\end{array}$ & $\begin{array}{l}\text { Bom Jardim da } \\
\text { Serra, SC }\end{array}$ & Eskuche 2007 & - & - & 28 & $\mathrm{Cfb}$ \\
\hline scobSC & Serra do Corvo Branco & Urubici, SC & Falkenberg 2003 & Expeditions & All fertile phanerogams & 83 & $\mathrm{Cfb}$ \\
\hline migrSC & Morro da Igreja & Urubici, SC & Falkenberg 2003 & Expeditions & All fertile phanerogams & 55 & $\mathrm{Cfb}$ \\
\hline srraSC & Serra do Rio do Rastro & $\begin{array}{l}\text { Bom Jardim da } \\
\text { Serra, SC }\end{array}$ & Falkenberg 2003 & Expeditions & All fertile phanerogams & 91 & $\mathrm{Cfb}$ \\
\hline $\operatorname{lagSC}$ & - & Lages, SC & Silva et al. (2012) & Plots & $\mathrm{DBH} \geq 5 \mathrm{~cm}$ & 86 & $\mathrm{Cfb}$ \\
\hline npRS & Fazenda Tupi & Nova Prata, RS & $\begin{array}{l}\text { Nascimento et al. } \\
2001\end{array}$ & Plots & $\mathrm{DBH} \geq 9,6 \mathrm{~cm}$ & 55 & $\mathrm{Cfb}$ \\
\hline $\operatorname{sfpRS}$ & $\begin{array}{l}\text { Floresta Nacional de São } \\
\text { Francisco de Paula }\end{array}$ & $\begin{array}{l}\text { São Francisco de } \\
\text { Paula, RS }\end{array}$ & $\begin{array}{l}\text { Rosário 2001; } \\
\text { Narvaes et al. } \\
\text { 2005; Duarte et } \\
\text { al. } 2006\end{array}$ & Plots & $\begin{array}{c}\text { Height } \geq 2 \mathrm{~m} ; \\
\mathrm{CBH} \geq 62.8 \mathrm{~cm} ; \mathrm{CBH} \geq \\
3 \mathrm{~cm} ; \\
\mathrm{DBH} \geq 5 \mathrm{~cm}\end{array}$ & 108 & $\mathrm{Cfb}$ \\
\hline bjRS & Rio Pelotas & Bom Jesus, RS & Brack et al. 2009 & Walking & - & 105 & $\mathrm{Cfb}$ \\
\hline cdsRS & Caxias do Sul & Caxias do Sul, RS & $\begin{array}{l}\text { Ramos \& Boldo } \\
2007\end{array}$ & Plots & - & 125 & $\mathrm{Cfb}$ \\
\hline vacRS & Refugiados & Vacaria, RS & Mauhs 2002 & Plots & Height $\geq 0.5 \mathrm{~m}$ & 57 & $\mathrm{Cfb}$ \\
\hline criRS & Criúva & Criúva, RS & $\begin{array}{l}\text { Rondon Neto et al. } \\
2002 \mathrm{~b}\end{array}$ & Plots & $\mathrm{DBH} \geq 5 \mathrm{~cm}$ & 37 & $\mathrm{Cfb}$ \\
\hline seroSC & Serra da Rocinha & $\begin{array}{l}\text { São José dos } \\
\text { Ausentes, RS }\end{array}$ & Falkenberg 2003 & Expeditions & All fertile phanerogams & 70 & $\mathrm{Cfb}$ \\
\hline
\end{tabular}

and spatial variables, we incorporated data concerning the altitude, climate (1960-1990), and geographical coordinates of each area. The geographic coordinates of the areas were based on the publications cited earlier. Altitudinal and climatic data were obtained from the WORLDCLIM databank (Hijmans et al. 2005), including: altitude (alt), Annual mean temperature (b1), Mean diurnal range (Mean of monthly (max temp - min temp)) (b2), Isothermality (b2/ b7) (x100) (b3), Temperature seasonality (standard deviation x100) (b4), Max temperature of warmest month (b5), Min temperature of coldest month (b6), Temperature annual range (b5-b6) (b7), Mean temperature of wettest quarter (b8), Mean temperature of driest quarter (b9), Mean temperature of warmest quarter (b10), Mean temperature of coldest quarter (b11), Annual precipitation (b12), Precipitation of wettest month (b13), Precipitation of driest month (b14), Precipitation seasonality (coefficient of variation) (b15), Precipitation of wettest quarter (b16), Precipitation of driest quarter (b17), Precipitation of warmest quarter (b18), and Precipitation of coldest quarter (b19).

\section{Data analysis}

Both the numbers of shared and exclusive species at the Campos Novos, Lages, and Painel sites were demonstrated using a Venn diagram. In order to evaluate the floristic relationships of these areas with other remnant $\mathrm{AF}$ sites in southern Brazil, a dendrogram displaying the hierarchical clustering of these areas was prepared utilizing the Sorensen coefficient as a measure of distance, and the UPGMA linking method as the cluster algorithm.

In order to determine the influence of environmental and spatial variables on the floristic patterns encountered, we employed NMDS (Non-metric multidimensional scaling) multivariate analysis with two dimensions $(\mathrm{k}=2)$ (Minchin 1987), and utilized the Sorensen coefficient as a measure of similarity. Although a common practice, we did not exclude rare species from these analyses as they have little influence in NMDS (Muotka et al. 2002). The adequacy of the ordination for interpretation was evaluated using a "stress" value. The variations explained by the ordination axes were calculated from nonlinear adjustments between the distances of the area as defined in the ordination as well as the original dissimilarity in values between the areas (Oksanen 2011). The significance of the "stress" value encountered was evaluated according to the methodology proposed by McCune \& Grace (2002) using the Monte Carlo test with 100 permutations. The environmental and spatial variables were adjusted a posteriori and inserted in the form of vectors into the ordination produced by NMDS, using 1000 permutations and excluding the redundant variables or those with low significance in the analysis $(P<0.001)$.

All of the analyses were made using the $\mathrm{R}$ statistical program (R Development Core Team 2010). The Limma package (Gentleman et al. 2004) was used to create the Venn diagram and the Vegan (Oksanen et al. 2009) and Cluster (Maechler et al. 2011) packages were used for multivariate analyses. 


\section{RESULTS}

\section{Species composition and richness}

A total of 147 species, 93 genera and 53 plant families were encountered in the three study fragments (table 2). Eight species were identified only to the family level (four species in Campos Novos, three species in Lages, and one species in Painel) and four only to the genus level (one species each in Campos Novos and Lages and two species in Painel); eight taxa were not identified at all due to the impossibility of making collections (two in Campos Novos, three in Lages, and three in Painel).

The families that most contributed to species richness in the three remnant areas evaluated were Myrtaceae (26 species), Fabaceae (12), Asteraceae (8), Lauraceae (8), Salicaceae (7), Solanaceae (7), and Aquifoliaceae (6). Together, these families comprised $50.68 \%$ of the total number of species identified. The richest genera were Solanum (7 species), Myrcia (7), Ilex (6), Eugenia (5), Myrceugenia (5), Maytenus (4), and Ocotea (4). Myrtaceae stood out as the family with the greatest species richness in all the three areas

Table 2. Floristic list of the tree species sampled in Araucaria Forest fragments located in the municipalities of Campos Novos (1), Lages (2), and Painel (3) on the Santa Catarina Plateau in southern Brazil. $\mathrm{M} / \mathrm{N}=$ municipalities where the specimens were collected (1,2 and 3) followed by their identification numbers at the LUSC Herbarium; specimens without identification numbers were not incorporated into the herbarium.

\begin{tabular}{|c|c|c|}
\hline Families & Species & $\mathrm{M} / \mathrm{N}$ \\
\hline Adoxaceae & Sambucus australis Cham. \& Schltdl. & $1(\mathrm{D} 2494)$ \\
\hline \multirow[t]{4}{*}{ Anacardiaceae } & Lithraea brasiliensis Marchand & 1 (D2495), 2 (D2594) \\
\hline & Schinus lentiscifolius Marchand & 2 \\
\hline & Schinus polygamus (Cav.) Cabrera & 2 (D2596), 3 (D2554) \\
\hline & Schinus terebinthifolius Raddi & 2 (D2595) \\
\hline \multirow[t]{2}{*}{ Annonaceae } & Annona rugulosa (Schltdl.) H.Rainer & 1 (D2496), 2 (D2597) \\
\hline & Annona sylvatica A.St.-Hil. & 1 \\
\hline Apocynaceae & Aspidosperma australe Müll.Arg. & 1 \\
\hline \multirow[t]{6}{*}{ Aquifoliaceae } & Ilex brevicuspis Reissek & 2 \\
\hline & Ilex dumosa Reissek & 1 (D2497), 2 (D2598) \\
\hline & Ilex microdonta Reissek & 2 (D2599), 3 (D2555) \\
\hline & Ilex paraguariensis A.St.-Hil. & 2 (D2600), 3 (D2556) \\
\hline & Ilex taubertiana Loes. & $3(\mathrm{D} 2557)$ \\
\hline & Ilex theezans Mart. ex Reissek & 2 (D2601), 3 (D2558) \\
\hline Araliaceae & Oreopanax fulvus Marchal & $1,2(\mathrm{D} 2602)$ \\
\hline Araucariaceae & Araucaria angustifolia (Bertol.) Kuntze & $1,2,3(\mathrm{D} 2559)$ \\
\hline Arecaceae & Syagrus romanzoffiana (Cham.) Glassman & 1 \\
\hline Asparagaceae & Cordyline spectabilis Kunth \& Bouché & $1(\mathrm{D} 2498)$ \\
\hline \multirow[t]{8}{*}{ Asteraceae } & Baccharis caprariifolia DC. & 3 \\
\hline & Baccharis dentata (Vell.) G.Barroso & 3 \\
\hline & Dasyphyllum spinescens (Less.) Cabrera & 2,3 \\
\hline & Dasyphyllum tomentosum (Spreng.) Cabrera & $2(\mathrm{D} 2603)$ \\
\hline & Gochnatia polymorpha (Less.) Cabrera & $2(\mathrm{D} 2604)$ \\
\hline & Piptocarpha angustifolia Dusén & 1,3 \\
\hline & Symphyopappus itatiayensis (Hieron.) R.M.King \& H.Rob. & 3 \\
\hline & Vernonanthura discolor (Spreng.) H.Rob. & 1 (D2499), 2 (D2605), 3 (D2560) \\
\hline Berberidaceae & Berberis laurina Billb. & 2 (D2606), 3 (D2561) \\
\hline \multirow[t]{2}{*}{ Bignoniaceae } & Handroanthus albus (Cham.) Mattos & $2(\mathrm{D} 2607)$ \\
\hline & Jacaranda puberula Cham. & 1 (D2500), 2 (D2608) \\
\hline Canellaceae & Cinnamodendron dinisii Schwacke & 1 (D2501), 2 (D2609) \\
\hline \multirow[t]{2}{*}{ Cannabaceae } & Celtis iguanaea (Jacq.) Sarg. & $2(\mathrm{D} 2610)$ \\
\hline & Trema micrantha (L.) Blume & 1 \\
\hline Cardiopteridaceae & Citronella paniculata (Mart.) R.A.Howard & 1 (D2502), 2 (D2611) \\
\hline
\end{tabular}


continuation

\begin{tabular}{|c|c|c|}
\hline Families & Species & $\mathrm{M} / \mathrm{N}$ \\
\hline \multirow[t]{5}{*}{ Celastraceae } & Maytenus aquifolia Mart. & $1(\mathrm{D} 2503)$ \\
\hline & Maytenus boaria Molina & $2(\mathrm{D} 2612)$ \\
\hline & Maytenus dasyclada Mart. & $2(\mathrm{D} 2613)$ \\
\hline & Maytenus ilicifolia Mart. ex Reissek & $3(\mathrm{D} 2562)$ \\
\hline & Schaefferia argentinensis Speg. & 1 (D2504) \\
\hline Clethraceae & Clethra scabra Pers. & 1 (D2505), 2 (D2614), 3 (D2563) \\
\hline Cordiaceae & Cordia americana (L.) Gottschling \& J.J.Mill. & $1(\mathrm{D} 2506)$ \\
\hline Cunoniaceae & Lamanonia ternata Vell. & 1 (D2507), 2 (D2615) \\
\hline Cyatheaceae & Cyathea delgadii Sternb. & $1(\mathrm{D} 2508)$ \\
\hline Dicksoniaceae & Dicksonia sellowiana Hook. & 1 (D2509), 2, 3 (D2564) \\
\hline Elaeocarpaceae & Sloanea monosperma Vell. & $1(\mathrm{D} 2510)$ \\
\hline Erythroxylaceae & Erythroxylum deciduum A.St.-Hil. & $2(\mathrm{D} 2616)$ \\
\hline Escalloniaceae & Escallonia bifida Link \& Otto & 2 (D2617) \\
\hline \multirow[t]{4}{*}{ Euphorbiaceae } & Gymnanthes concolor (Spreng.) Müll.Arg. & $1(\mathrm{D} 2511)$ \\
\hline & Sapium glandulosum (L.) Morong & 1,2 \\
\hline & Sebastiania brasiliensis Spreng. & 1 (D2512), 2 (D2618) \\
\hline & Sebastiania commersoniana (Baill.) L.B.Sm. \& Downs & $1,2(\mathrm{D} 2619)$ \\
\hline \multirow[t]{6}{*}{ Fabaceae Faboideae } & Dalbergia frutescens (Vell.) Britton & 1 (D2513), 2 (D2620) \\
\hline & Erythrina falcata Benth. & 1 \\
\hline & Lonchocarpus campestris Mart. ex Benth. & 1 \\
\hline & Machaerium paraguariense Hassl. & $2(\mathrm{D} 2621)$ \\
\hline & Machaerium stipitatum (DC.) Vogel & $2(\mathrm{D} 2622)$ \\
\hline & Myrocarpus frondosus Allemão & 1 \\
\hline \multirow[t]{6}{*}{ Fabaceae Mimosoideae } & Albizia niopoides (Spruce ex Benth.) Burkart & 1 \\
\hline & Inga lentiscifolia Benth. & $3(\mathrm{D} 2565)$ \\
\hline & Inga sessilis (Vell.) Mart. & $2(\mathrm{D} 2623)$ \\
\hline & Inga striata Benth. & $1(\mathrm{D} 2514)$ \\
\hline & Mimosa scabrella Benth. & $1,2,3(\mathrm{D} 2566)$ \\
\hline & Parapiptadenia rigida (Benth.) Brenan & $1(\mathrm{D} 2515)$ \\
\hline Lamiaceae & Vitex megapotamica (Spreng.) Moldenke & $1(\mathrm{D} 2516)$ \\
\hline \multirow[t]{8}{*}{ Lauraceae } & Cinnamomum атоепит (Nees \& Mart.) Kosterm. & 2 (D2624), 3 (D2567) \\
\hline & Nectandra lanceolata Nees & 2 \\
\hline & Nectandra megapotamica (Spreng.) Mez & 1 (D2517), 2 \\
\hline & Ocotea diospyrifolia (Meisn.) Mez & 2 \\
\hline & Ocotea porosa (Nees \& Mart.) Barroso & 1 (D2518) \\
\hline & Ocotea puberula (Rich.) Nees & 2 \\
\hline & Ocotea pulchella Mart. & 1 (D2519), 2 (D2625), 3 (D2568) \\
\hline & Persea willdenowii Kosterm. & 1 (D2520), 3 (D2569) \\
\hline Malvaceae & Luehea divaricata Mart. & $1(\mathrm{D} 2521)$ \\
\hline \multirow[t]{2}{*}{ Melastomataceae } & Miconia hiemalis A.St.-Hil. \& Naudin ex Naudin & 2 (D2626) \\
\hline & Miconia cinerascens Miq. & 1 (D2522) \\
\hline \multirow[t]{4}{*}{ Meliaceae } & Cabralea canjerana (Vell.) Mart. & 1,2 \\
\hline & Cedrela fissilis Vell. & 1,2 (D2627) \\
\hline & Trichilia claussenii C.DC. & $1(\mathrm{D} 2523)$ \\
\hline & Trichilia elegans A.Juss. & $1(\mathrm{D} 2524)$ \\
\hline \multirow[t]{7}{*}{ Myrtaceae } & Acca sellowiana (O.Berg) Burret & 2 (D2628), 3 (D2570) \\
\hline & Blepharocalyx salicifolius (Kunth) O.Berg & 2 (D2629), 3 (D2571) \\
\hline & Calyptranthes concinna DC. & 2 (D2630), 3 (D2572) \\
\hline & Campomanesia guazumifolia (Cambess.) O.Berg & 1 \\
\hline & Campomanesia xanthocarpa O.Berg & 1,2 (D2631), 3 \\
\hline & Eugenia pluriflora DC. & $2(\mathrm{D} 2632)$ \\
\hline & Eugenia pyriformis Cambess. & 2 (D2633) \\
\hline
\end{tabular}


continuation

\begin{tabular}{|c|c|c|}
\hline Families & Species & $\mathrm{M} / \mathrm{N}$ \\
\hline & Eugenia ramboi D.Legrand & 1 \\
\hline & Eugenia uniflora L. & 1 (D2525), 2 (D2634) \\
\hline & Eugenia uruguayensis Cambess. & $2(\mathrm{D} 2635)$ \\
\hline & Myrceugenia euosma (O.Berg) D.Legrand & 2 (D2636), 3 (D2573) \\
\hline & Myrceugenia glaucescens (Cambess.) D.Legrand \& Kausel & 3 \\
\hline & Myrceugenia myrcioides (Cambess.) O.Berg & 2 (D2637), 3 (D2574) \\
\hline & Myrceugenia ovata (Hook. \& Arn.) O.Berg & $2(\mathrm{D} 2638), 3(\mathrm{D} 2575)$ \\
\hline & Myrceugenia oxysepala (Burret) D.Legrand \& Kausel & $3(\mathrm{D} 2576)$ \\
\hline & Myrcia bombycina (O.Berg) Nied. & 1 \\
\hline & Myrcia guianensis (Aubl.) DC. & 1 (D2640), 2 \\
\hline & Myrcia hartwegiana (O.Berg) Kiaersk. & 3 \\
\hline & Myrcia hatschbachii D.Legrand & 2 \\
\hline & Myrcia laruotteana Cambess. & $2(\mathrm{D} 2641)$ \\
\hline & Myrcia multiflora (Lam.) DC. & 2 \\
\hline & Myrcia palustris DC. & $2(\mathrm{D} 2642)$ \\
\hline & Myrcianthes gigantea (D.Legrand) D.Legrand & 1,2 (D2643) \\
\hline & Myrcianthes pungens (O.Berg) D.Legrand & 1 \\
\hline & Myrrhinium atropurpureum Schott & 2 (D2644), 3 (D2577) \\
\hline & Plinia trunciflora (O.Berg) Kausel & 1 \\
\hline Phytolaccaceae & Seguieria americana $\mathrm{L}$. & 1 \\
\hline Picramniaceae & Picramnia parvifolia Engl. & $1(\mathrm{D} 2526)$ \\
\hline Podocarpaceae & Podocarpus lambertii Klotzsch ex Endl. & $2(\mathrm{D} 2645)$ \\
\hline Polygonaceae & Ruprechtia laxiflora Meisn. & $1(\mathrm{D} 2527)$ \\
\hline \multirow[t]{3}{*}{ Primulaceae } & Myrsine coriacea (Sw.) Roem. \& Schult. & 1 (D2528), 2 (D2646), 3 (D2578) \\
\hline & Myrsine guianensis (Aubl.) Kuntze & $1(\mathrm{D} 2529)$ \\
\hline & Myrsine umbellata Mart. & 1 (D2530), 2 (D2647) \\
\hline Proteaceae & Roupala montana Aubl. & 1 (D2531), 2 (D2648), 3 (D2579) \\
\hline Quillajaceae & Quillaja brasiliensis (A.St.-Hil. \& Tul.) Mart. & $2(\mathrm{D} 2649)$ \\
\hline \multirow[t]{2}{*}{ Rhamnaceae } & Rhamnus sphaerosperma $\mathrm{Sw}$. & 2 (D2650), 3 (D2580) \\
\hline & Scutia buxifolia Reissek & $2(\mathrm{D} 2651)$ \\
\hline Rosaceae & Prunus myrtifolia (L.) Urb. & 1 (D2532), 2 (D2652), 3 (D2581) \\
\hline \multirow[t]{3}{*}{ Rubiaceae } & Chomelia obtusa Cham. \& Schltdl. & $1(\mathrm{D} 2533)$ \\
\hline & Cordiera concolor (Cham.) Kuntze & 1 (D2534) \\
\hline & Coutarea hexandra (Jacq.) K.Schum. & 1 (D2535), 2 (D2653) \\
\hline \multirow[t]{4}{*}{ Rutaceae } & Helietta apiculata Benth. & $1(\mathrm{D} 2536)$ \\
\hline & Zanthoxylum fagara (L.) Sarg. & $1(\mathrm{D} 2537)$ \\
\hline & Zanthoxylum kleinii (R.S.Cowan) P.G.Waterman & 1 (D2538), 2 (D2654), 3 (D2582) \\
\hline & Zanthoxylum rhoifolium Lam. & 1,2 (D2655), 3 (D2583) \\
\hline \multirow[t]{7}{*}{ Salicaceae } & Banara parviflora (A.Gray) Benth. & $1(\mathrm{D} 2539)$ \\
\hline & Banara tomentosa Clos & 1 (D2540), 2 (D2656) \\
\hline & Casearia decandra Jacq. & 1 (D2541), 2 (D2657) \\
\hline & Casearia obliqua Spreng. & $2(\mathrm{D} 2658)$ \\
\hline & Casearia sylvestris $\mathrm{Sw}$. & $1(\mathrm{D} 2542)$ \\
\hline & Xylosma ciliatifolia (Clos) Eichler & 1,2 (D2659), 3 \\
\hline & Xylosma tweediana (Clos) Eichler & 1 (D2543), 2 (D2660) \\
\hline \multirow[t]{4}{*}{ Sapindaceae } & Allophylus edulis (A.St.-Hil. et al.) Radlk. & 1 (D2544), 2 (D2661) \\
\hline & Allophylus guaraniticus (A.St.-Hil.) Radlk. & 1 (D2545), 2 (D2662), 3 (D2584) \\
\hline & Cupania vernalis Cambess. & 1 (D2546), 2 (D2663) \\
\hline & Matayba elaeagnoides Radlk. & 1 (D2547), 2 (D2664) \\
\hline Sapotaceae & Chrysophyllum marginatum (Hook. \& Arn.) Radlk. & $1(\mathrm{D} 2548)$ \\
\hline \multirow{2}{*}{ Solanaceae } & Solanum mauritianum Scop. & $3(\mathrm{D} 2585)$ \\
\hline & Solanum pabstii L.B.Sm. \& Downs & 2 (D2665), 3 (D2586) \\
\hline
\end{tabular}


continuation

\begin{tabular}{|c|c|c|}
\hline Families & Species & $\mathrm{M} / \mathrm{N}$ \\
\hline & Solanum paranense Dusén & $3(\mathrm{D} 2587)$ \\
\hline & Solanum pseudoquina A.St.-Hil. & 1 (D2549), 3 (D2588) \\
\hline & Solanum ramulosum Sendtn. & $3(\mathrm{D} 2589)$ \\
\hline & Solanum sanctaecatharinae Dunal & 1 (D2550), 2 (D2666), 3 (D2590) \\
\hline & Solanum variabile Mart. & $3(\mathrm{D} 2591)$ \\
\hline Styracaceae & Styrax leprosus Hook. \& Arn. & 1 (D2551), 2 (D2667) \\
\hline Symplocaceae & Symplocos uniflora (Pohl) Benth. & 1 (D2552), 2 (D2668) \\
\hline Thymelaeaceae & Daphnopsis racemosa Griseb. & 2 (D2669), 3 (D2592) \\
\hline Verbenaceae & Duranta vestita Cham. & $2(\mathrm{D} 2670)$ \\
\hline Winteraceae & Drimys brasiliensis Miers & 1 (D2553), 2 (D2671), 3 (D2593) \\
\hline
\end{tabular}

studied. The families that most contributed to arboreal floristic richness in Campos Novos were Myrtaceae ( 9 species), Fabaceae (8), Salicaceae (6), Euphorbiaceae (4), Lauraceae (4), Meliaceae (4), Rutaceae (4), and Sapindaceae (4); the species distributions among these different genera were quite homogeneous, although Myrsine stood out with three species. The families that most contributed to species richness in Lages were Myrtaceae (18 species), Lauraceae (6), Aquifoliaceae (5), Salicaceae (5), Anacardiaceae (4), Asteraceae (4), and Sapindaceae (4); the most important genera were Myrcia (5 species), Ilex (5), Eugenia (4), Myrceugenia (3), Ocotea (3), and Schinus (3). The families that most contributed to species richness in Painel were Myrtaceae (11 species), Solanaceae (7), Asteraceae (6), Aquifoliaceae (4), and Lauraceae (3); the richest genera were Solanum (7 species), Myrceugenia (5), and Ilex (4).

The species richnesses values in the remnant forest areas at Campos Novos, Lages, and Painel were 87, 91 and 49 respectively (figure 2). Only a low percentage of species were shared among all three sample areas $(10.88 \%)$. The site with the greatest proportion of exclusive species was Campos Novos (45.98\%), while Painel had the lowest (26.53\%); the latter site shared $67.35 \%$ of its species with Lages. Of the total number species encountered in Lages, $48.35 \%$ were shared with Campos Novos, $36.26 \%$ with Painel, while $32.97 \%$ were exclusive.

\section{Phytogeography}

Examinations of the AF fragments in southern Brazil indicated the existence of three large floristic groups (figure 3). Group 1 was composed of high-montane areas in the states of Santa Catarina and Rio Grande do Sul; Group 2 was formed by forest remnants in Paraná State in the Paraná River basin (Arapoti, Tibagi, and Irati); and

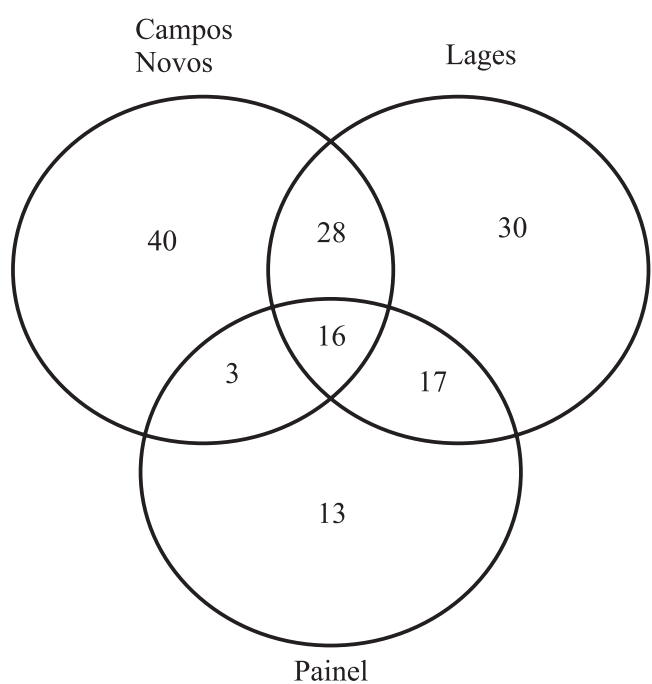

Figure 2. Venn diagram with species distributions in Araucaria Forest fragments located in the municipalities of Campos Novos, Lages, and Painel in Santa Catarina State, Brazil.

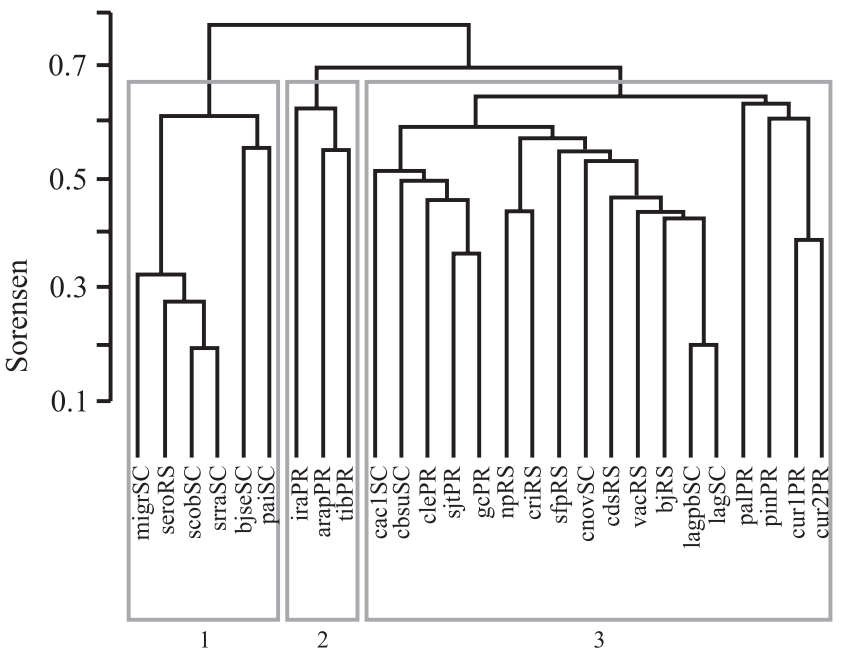

Figure 3. Dendrogram produced by cluster analysis of the Araucaria Forest remnants examined in southern Brazil, using the Sorensen coefficient is a measure of distance and UPGMA as the clustering algorithm. See table 1 for the locality names. 
Group 3 was formed by a large number of remnant areas distributed among three states located in the hydrographic basins of the Paraná and Uruguay rivers and those rivers that drain directly into the southern Atlantic. The forest remnant located in the municipality of Painel was placed in Group 1 and was the most floristically distinct but showed greater floristic similarity with Bom Jardim da Serra, SC. The fragment area in Lages (Group 3) demonstrated greater floristic similarity with another fragment from the same municipality, and both of them demonstrated high similarity with Bom Jesus, Vacaria, Caxias do Sul, Campos Novos, and São Francisco de Paula - all of which are situated in the Upper-Uruguay region.

The "stress" value in the NMDS ordination analysis (figure 4) was $15.78 \%(P<0.01)$, which corresponds to an explained variance of $97.51 \%$ - indicating that the diagram was adequate for our interpretations. The floristic groups defined in the dendrogram are indicated in the NMDS diagram by continuous lines. The variables that demonstrated elevated significance $(P<0.001)$ (table 3$)$ within the ordination are represented by vectors. It can be seen that the variables that best explained the separation of the three groups were altitude (alt), Annual mean

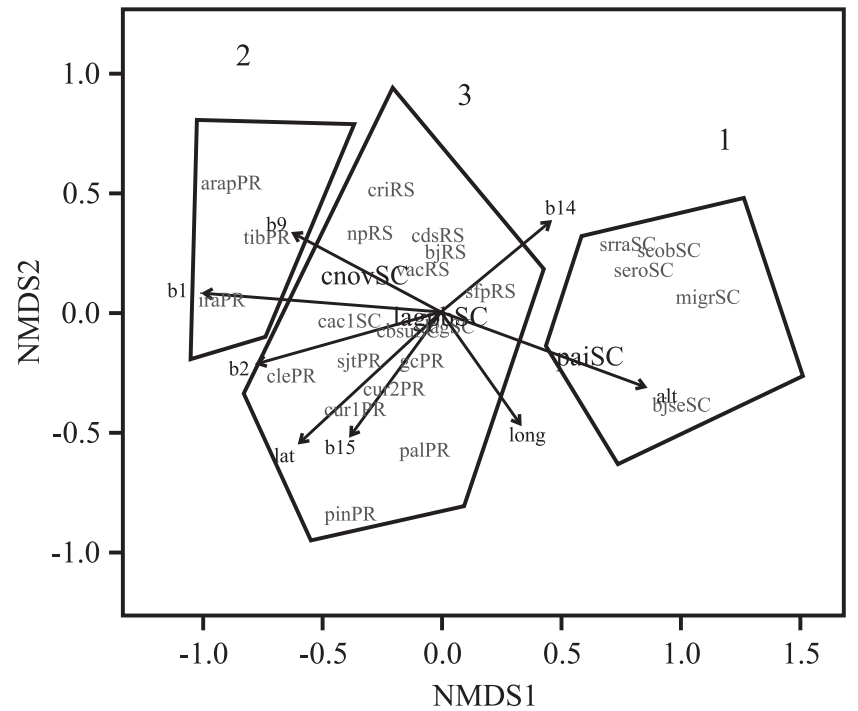

Figure 4. Ordination diagram produced by NMDS (Nonmetric multidimensional scaling) analysis of Araucaria Forest remnants located in southern Brazil. (Alt $=$ altitude; b1 = Annual mean temperature; $b 2=$ Mean diurnal temperature range; b9 = Mean temperature of driest quarter; b14 = Precipitation of driest month; b15 = Precipitation seasonality; lat $=$ latitude; long = longitude). See table 1 for the locality names.

Table 3. Environmental and spatial variables $(P<0.001)$ fitted to the ordination produced by NMDS of the Araucaria Forest fragments in southern Brazil. * = Identification codes for the areas; long $=$ longitude $\left({ }^{\circ}\right)$; lat $=$ latitude $\left({ }^{\circ}\right)$; alt $=$ altitude $(\mathrm{m})$; $\mathrm{b} 1=$ Mean annual temperature $\left({ }^{\circ} \mathrm{C}\right)$; b2 $=$ Mean daily thermal amplitude $\left({ }^{\circ} \mathrm{C}\right) ; \mathrm{b} 9=$ Mean temperature during the most humid trimester $\left({ }^{\circ} \mathrm{C}\right) ; \mathrm{b} 14=$ Precipitation during the driest month $(\mathrm{mm})$; b15 = Precipitation seasonality (variation coefficient). Acronyms for the Brazilian states: $\mathrm{RS}=$ Rio Grande do Sul; PR = Paraná; $\mathrm{SC}=$ Santa Catarina.

\begin{tabular}{|c|c|c|c|c|c|c|c|c|c|}
\hline \multirow{2}{*}{ Area* } & \multirow{2}{*}{ Municipality } & \multicolumn{8}{|c|}{ Geographical and climatic variables } \\
\hline & & long. (W) & lat. (S) & alt. & b1 & b2 & b9 & b14 & b15 \\
\hline arapPR & Arapoti, PR & 49.9605 & 23.8525 & 613 & 19.8 & 12.1 & 17.4 & 48 & 3.82 \\
\hline pinPR & Pinhais, PR & 49. 1305 & 25.4069 & 920 & 17.1 & 10.0 & 13.9 & 77 & 3.12 \\
\hline sjtPR & São João do Triunfo, PR & 50.0988 & 25.5717 & 891 & 17.1 & 11.7 & 13.3 & 78 & 2.92 \\
\hline Cur1PR & Curitiba, PR & 49. 2424 & 25.4592 & 907 & 17.2 & 10.2 & 14.3 & 75 & 2.97 \\
\hline Cur2PR & Curitiba, PR & 49. 2424 & 25.4592 & 907 & 17.2 & 10.2 & 14.3 & 75 & 2.97 \\
\hline iraPR & Irati, PR & 50.6307 & 25.4656 & 859 & 17.3 & 11.7 & 13.5 & 74 & 2.66 \\
\hline tibPR & Tibagi, PR & 50.4166 & 24.516 & 764 & 18.5 & 12.7 & 14.9 & 54 & 2.88 \\
\hline clePR & Clevelândia, PR & 52.2275 & 26.3317 & 939 & 16.5 & 13.3 & 16.4 & 130 & 1.9 \\
\hline gcPR & General Carneiro, PR & 51.4247 & 26.4369 & 1162 & 15.3 & 12.3 & 11.4 & 112 & 1.39 \\
\hline palPR & Palmeira, PR & 50.0260 & 25.6033 & 808 & 17.4 & 11.7 & 13.5 & 74 & 2.96 \\
\hline lagpbSC & Lages, SC & 50.1882 & 27.9162 & 993 & 16.1 & 10.5 & 12.4 & 99 & 1.61 \\
\hline paiSC & Painel, SC & 50.1049 & 27.9289 & 1196 & 15.0 & 10.2 & 12.5 & 105 & 1.55 \\
\hline cnovSC & Campos Novos, SC & 51.1808 & 27.6021 & 711 & 17.0 & 11.1 & 17 & 112 & 1.31 \\
\hline $\operatorname{cacSC}$ & Caçador, SC & 51.0166 & 26.7833 & 934 & 16.3 & 12.1 & 12.1 & 117 & 1.19 \\
\hline cbsuSC & Campo Belo do Sul, SC & 50.8166 & 28.0001 & 956 & 16.0 & 10.5 & 15.9 & 120 & 1.11 \\
\hline bjseSC & Bom Jardim da Serra, SC & 49.6249 & 28.3370 & 1319 & 13.9 & 9.5 & 11.3 & 112 & 1.62 \\
\hline scobSC & Urubici, SC & 49.3663 & 28.0575 & 1491 & 12.9 & 9.4 & 10.6 & 115 & 1.75 \\
\hline migrSC & Urubici, SC & 49.4832 & 28.1333 & 1501 & 12.9 & 9.4 & 10.5 & 115 & 1.66 \\
\hline
\end{tabular}




\begin{tabular}{|c|c|c|c|c|c|c|c|c|c|}
\hline \multirow{2}{*}{ Area* } & \multirow{2}{*}{ Municipality } & \multicolumn{8}{|c|}{ Geographical and climatic variables } \\
\hline & & long. (W) & lat. (S) & alt. & b1 & b2 & b9 & b14 & b15 \\
\hline srraSC & Bom Jardim da Serra, SC & 49.5499 & 28.4001 & 1329 & 13.7 & 9.4 & 11.2 & 113 & 1.60 \\
\hline $\operatorname{lagSC}$ & Lages, SC & 50.3211 & 27.8534 & 916 & 16.5 & 10.6 & 12.8 & 95 & 1.61 \\
\hline $\mathrm{npRS}$ & Nova Prata, RS & 51.8839 & 28.9324 & 395 & 18.4 & 10 & 14.5 & 112 & 1.07 \\
\hline sfpRS & São Francisco de Paula, RS & 50.3833 & 29.4501 & 814 & 15.5 & 9.5 & 16.7 & 137 & 0.90 \\
\hline bjRS & Bom Jesus, RS & 50.6132 & 28.4128 & 866 & 16.4 & 10 & 13.8 & 114 & 1.25 \\
\hline cdsRS & Caxias do Sul, RS & 51.2500 & 28.7500 & 786 & 16.9 & 10.4 & 18.7 & 125 & 1.25 \\
\hline vacRS & Vacaria, RS & 50.9333 & 28.6668 & 843 & 16.5 & 9.9 & 13.9 & 127 & 1.11 \\
\hline criRS & Criúva, RS & 50.9303 & 29.0047 & 856 & 16.3 & 9.6 & 13.7 & 133 & 1.14 \\
\hline seroRS & São José dos Ausentes, RS & 49.9499 & 28.7834 & 1187 & 14.3 & 9.5 & 11.8 & 122 & 1.31 \\
\hline
\end{tabular}

temperature (b1), and Mean temperature of driest quarter (b9). The extremes of this gradient are represented by the areas in Group 1 (with the highest and coldest sites) and by the areas of Group 2 (with the lowest altitude and warmest sites). Internally within Group 3 (which had the largest area), the greatest species substitution occurred along an environmental gradient associated with latitude (lat), Precipitation seasonality (coefficient of variation) (b15), and Precipitation of driest month (b14).

\section{DISCUSSION}

The numbers of species encountered in each fragment in the present study were indicative of low richness (in the case of Painel) as well as elevated richness (in the case of Lages and Campos Novos) in comparison with other studies undertaken in AF fragments in southern Brazil with values that varied between 134 in Arapoti and Palmeira (PR) to 26 in Clevelândia (PR) (see table 1). Myrtaceae, the plant family that most contributed to species richness in the three study fragments, had already been cited by Mori et al. (1983) as the richest family in different Atlantic Forest formations. Additionally, a number of studies undertaken in southern Brazil have confirmed important contributions of Myrtaceae to species richness (e.g. Jurinitz \& Jarenkow 2003).

The low percentages of species shared among the three fragments studied indicated that AFs are not floristically homogeneous formations. The ordination of the study areas and their relationships with the spatial and environmental variables examined demonstrated that the remnant $\mathrm{AF}$ areas have floristic variations that are associated with their distributions from West to East and North and South, reflecting the overall environmental heterogeneity of the region. It is worth noting that aspects related to historical perturbations in these areas also influence tree species distributions (even though this factor was not evaluated the present study) (Silva et al. 2009).

To the east, near the coast, there are important mountain chains formed by the Serra do Mar and Serra Geral mountains that constitute the highest elevations in southern Brazil. Heavy fogs frequently occur in these localities as well as snap freezes and frequent snowfalls, principally in the Serra Catarinense and Serra Gaúcha regions. This is where the areas forming floristic Group 1 are found, including the present study fragment in the municipality of Painel. A number of species encountered in this fragment were exclusive to Lages and Campos Novos, principally those belonging to the genera Ilex, Baccharis, Symphyopappus, Myrceugenia, and Solanum that contain high-altitude indicator species. The genera Drimys and Clethra which Webster (1995) identified as typical of Neotropical cloud-forest areas also occur in this fragment. The low richness observed in Painel might be a result of the high rigor and selectivity of the climatic conditions in that area.

Land elevation gradually diminishes westward as the principal watercourses in the region drain towards the Paraná and Uruguay rivers. The AF accompany the principle hydrographic basins forming these rivers, from the highest elevations to altitudes near $500 \mathrm{~m}$ (where the climate becomes warmer and there are stronger influences of typical components of seasonal forests). The area near Campos Novos, along the Canoas River (which together with the Pelotas River forms the Uruguay River), is an example of this situation, with a significant proportion of its species being exclusive in relation to those seen at Lages and Painel, including: Schaefferia argentinensis, Cordia americana, Myrocarpus frondosus, and Parapiptadenia rigida - all typical of Seasonal Deciduous Forests of the Upper-Uruguay river basin.

The influence of altitudinal gradients on the floristic patterns of neotropical forests has been demonstrated 
by a number of authors (e.g. Oliveira Filho et al. 1994, 2005, Oliveira Filho \& Fontes 2000, Scudeller et al. 2001) and was shown to be (together with temperature) one of the principal variables that influenced species distributions in the three area studied here. As was noted by Oliveira Filho \& Fontes (2000), various environmental changes occur along this gradient - such as temperature variations and the occurrence of freezing events - which can explain the strong changes observed in species distributions. Considering the three areas evaluated in the present study, Campos Novos is positioned at one of the extremes of this gradient at an altitude of $700 \mathrm{~m}$ and Painel at the other $(1196 \mathrm{~m})$. Both of these areas share large numbers of species with the Lages site (993 m), which is geographically situated between the two extremes and can be considered a transition area.

Passing through states of Santa Catarina to Rio Grande do Sul from north to south, there are two large hydrographic basins that together form the Platina Basin (the Paraná and Uruguay river basins). Even though this region does not experience a well-defined dry season, our results demonstrated an environmental gradient between these two basins associated principally with the precipitation regime (b14 and b15) (see figure 3). These results corroborate observations made by Klein (1978), who classified the AFs in Santa Catarina State in terms of their hydrographic basins. Authors such as Silva et al. (2006) likewise observed the tendency of areas that occur in the same hydrological basin to cluster in terms of their species compositions. One of the factors that could help explain the observed floristic compartmentalization in terms of hydrological basins would be the influence of riparian forests that function as dispersal corridors for arboreal species. This concept of tree species migration has been examined by a number of authors using different methodologies (e.g. Rambo 1951, Budke et al. 2004).

The Araucaria forests analyzed here could be classified into three distinct phytogeographical units: i) high altitude areas with lower mean annual temperatures and influences of cloud/fog cover (where the Painel site was established); ii) lower altitude areas with greater mean annual temperatures, situated in the Paraná River basin; iii) areas situated in the Paraná and UpperUruguay river basins as well as the smaller river basins that drain directly into the southern Atlantic (the Campos Novos and Lages sites). The environmental variables most closely correlated with the high levels of species substitutions among three sites were altitude, mean annual temperature, and mean temperature of driest quarter.
Acknowledgments - The authors thank CNPq for financial support through Edital MCT/CNPq 15/2007, which allowed us to undertake this study; the Brochmann Polis - Industrial and Florestal S/A company for allowing us access to their property at Campos Novos; and Tio Deja and Sr. Jorge Gamborgi for graciously allowing us access to their properties in Painel and Lages respectively.

\section{REFERENCES}

APG III. 2009. An update of the Angiosperm Phylogeny Group classification for the orders and families of flowering plants: APG III. Botanical Journal of the Linnean Society 161:105-121.

Behling H, Pillar V. 2007. Late Quaternary vegetation, biodiversity and fire dynamics on the southern Brazilian highland and their implication for conservation and management of modern Araucaria forest and grassland ecosystems. Philosophical Transactions of the Royal Society B 362:243-251.

Blum CT, Silva DAT, Hase LM, Miranda DLC. 2003. Caracterização florística e ecológica de remanescentes florestais no Rio das Cinzas, Norte Pioneiro, PR. In Anais do I Seminário Nacional de Degradação e Recuperação Ambiental. Sobrade, Foz do Iguaçu.

Brack P, Singer RF, Casagrande A, Pedrollo CT, Milanesi LS, Grings M, Panizzi R, Talbot V. 2009. Levantamento preliminar da flora e da vegetação do vale do rio Pelotas, no município de Bom Jesus, RS, e a importância de sua conservação. INGA Estudo Ambientais. http://migre. $\mathrm{me} / \mathrm{xHPc}$ (accessed $2010 \mathrm{Mar}$ ).

Budke JC, Giehl ELH, Athayde EA, Eisenger SM, Záchia RA. 2004. Florística e fitossociologia do componente arbóreo de uma floresta ribeirinha, arroio Passo das Tropas, Santa Maria, RS, Brasil. Acta Botanica Brasilica 18:581-589.

Dias M, Vieira A, Nakajima J, Pimenta J, Lobo P. 1998. Composição florística e fitossociologia do componente arbóreo das florestas ciliares do Rio Iapó, na bacia do rio Tibagi, Tibagi, PR. Revista Brasileira de Botânica 21:183-195.

Duarte L, Machado R, Hartz S, Pillar V, Bruelheide H. 2006. What saplings can tell us about forest expansion over natural grasslands. Journal of Vegetation Science 17:799-808.

Eskuche U. 2007. El bosque de Araucaria con Podocarpus y los campos de Bom Jardim da Serra, Santa Catarina (Brasil meridional). Boletim de la Sociedad Argentina de Botánica 42:295-308.

Falkenberg D. 2003. Matinhas nebulares e vegetação rupícola dos Aparados da Serra Geral (SC/RS), sul do Brasil. Tese de doutorado, Universidade Estadual de Campinas, Campinas.

Formento S, Schorn L, Ramos R. 2004. Dinâmica estrutural arbórea de uma Floresta Ombrófila Mista em Campo Belo do Sul, SC. Cerne 10:196-212. 
Gentleman R, Carey V, Bates D, Bolstad B, Dettling M, Dudoit S, Ellis B, Gautier L, Ge Y, Gentry J. 2004. Bioconductor: open software development for computational biology and bioinformatics. Genome Biology 5:R80.

Gomes M, Filizola H, Spadotto C. 2006. Classificação das áreas de recarga do sistema aqǘf́ero guarani no Brasil em domínios pedomorfoagroclimáticos - subsídio aos estudos de avaliação de risco de contaminação das águas subterrâneas. Revista do Departamento de Geografia 18:67-74.

Hijmans R, Cameron S, Parra J, Jones P, Jarvis A. 2005. Very high resolution interpolated climate surfaces for global land areas. International Journal of Climatology 25:1965-1978.

IBGE. 1992. Manual técnico da vegetação brasileira. Série: Manuais técnicos em geociências n.1. Fundação Instituto Brasileiro de Geografia e Estatística, Rio de Janeiro.

Iurk MC. 2008. Levantamento florístico de um fragmento de Floresta Ombrófila Mista Aluvial do Rio Iguaçu, município de Palmeira - PR. Dissertação de mestrado, Universidade Federal do Paraná, Curitiba.

Jurinitz C, Jarenkow J. 2003. Estrutura do componente arbóreo de uma floresta estacional na Serra do Sudeste, Rio Grande do Sul, Brasil. Revista Brasileira de Botânica 26:475-487.

Klein R. 1960. O aspecto dinâmico do pinheiro brasileiro. Sellowia 12:17-44.

Klein R. 1978. Mapa fitogeográfico do Estado de Santa Catarina. In Flora Ilustrada Catarinense (R. Reitz, ed.). Herbário Barbosa Rodrigues, Itajaí, Parte V, p.1-24.

Kozera C, Dittrich V, Silva S. 2006. Fitossociologia do componente arbóreo de um fragmento de Floresta Ombrófila Mista Montana, Curitiba, PR, BR. Floresta 36:225-237.

Leite P, Klein R. 1990. Vegetação. Geografia do Brasil Região Sul. Fundação Instituto Brasileiro de Geografia e Estatística, Rio de Janeiro.

Lingner DV, Oliveira YMM, Rosot NC, Dlugosz FL. 2007. Caracterização da estrutura e da dinâmica de um remanescente de Floresta com Araucária no Planalto Catarinense. Pesquisa Florestal Brasileira 55:55-66.

Maechler M, Rousseeuw P, Struyf A, Hubert M, Hornik K. 2011. Cluster: Cluster analysis basics and extensions. $\mathrm{R}$ package version 1.14.1.

Mauhs J. 2002. Fitossociologia e regeneração natural de um fragmento de Floresta Ombrófila Mista exposto a perturbações antrópicas. Dissertação de mestrado, Universidade do Vale dos Sinos, São Leopoldo.

Mccune B, Grace JB. 2002. Analysis of ecological communities. Mjm Software Design. Gleneden Beach.

Minchin P. 1987. An evaluation of the relative robustness of techniques for ecological ordination. Plant Ecology 69:89-107.

Mobot. Missouri Botanical Garden. 2008. http://www. tropicos.org/ (accessed 2010 Dec 15).
Mori S, Boom B, Carvalino A, Santos T. 1983. Ecological importance of Myrtaceae in an eastern Brazilian wet forest. Biotropica 15:68-70.

Muotka T, Paavola R, Haapala A, Novikmec M, Laasonen P. 2002. Long-term recovery of stream habitat structure and benthic invertebrate communities from in-stream restoration. Biological Conservation 105:243-253.

Narvaes I, Brena D, Longhi S. 2005. Estrutura da regeneração natural em Floresta Ombrófila Mista na Floresta Nacional de São Francisco de Paula, RS. Ciência Florestal 15:331-342.

Nascimento A, Longhi S, Brena D. 2001. Estrutura e padrões de distribuição espacial de espécies arbóreas em uma amostra de Floresta Ombrófila Mista em Nova Prata, RS. Ciência Florestal 11:105-119.

Negrelle R, Silva F. 1992. Fitossociologia de um trecho de floresta com Araucaria angustifolia (Bert.) O. Ktze. no município de Caçador-SC. Boletim de Pesquisa Florestal 24:37-54.

Oksanen J, Kindt R, Legendre P, O'Hara B, Simpson G, Stevens M. 2009. Vegan: community ecology package. $\mathrm{R}$ package version, v.1, p.8.

Oksanen J. 2011. Multivariate analysis of ecological communities in R: vegan tutorial. http://vegan.r-forge. r-project.org/ (accessed 2011 Feb 23).

Oliveira Filho AT, Vilela E, Carvalho D, Gavilanes M. 1994. Differentiation of streamside and upland vegetation in an area of montane semideciduous forest in southeastern Brazil. Flora 189:287-305.

Oliveira Filho AT, Fontes MAL. 2000. Patterns of floristic differentiation among Atlantic forests in south-eastern Brazil, and the influence of climate Biotropica 32: 793-810.

Oliveira Filho AT, Tameirão Neto E, Carvalho WAC, Werneck M, Brina AE, Vida CV, Rezende SC, Pereira JA. 2005. Análise do compartimento arbóreo de áreas de floresta atlântica senso lato na região das bacias do leste (Bahia, Minas Gerais, Espírito Santo e Rio de Janeiro). Rodriguésia 56:185-235.

R Development Core Team. 2010. R: A language and environment for statistical computing. R Foundation for Statistical Computing. http://www.R-project.org (accessed Mar 2010).

Rambo B. 1951. A imigração da selva higrófila no Rio Grande do Sul. Anais Botânicos do Herbário Barbosa Rodrigues 3:55-91.

Ramos AJK, Boldo E. 2007. Diversidade florística e aspectos fitossociológicos de formações florestais em estágio sucessional secundário na Floresta Ombrófila Mista, Município de Caxias do Sul - RS. Revista Brasileira de Agroecologia 2:111-116.

Rondon Neto RM, Kozera C, Andrade R, Cecy A, Hummes A, Fritzsons E, Caldeira M, Maciel N, Souza M. 2002a. Caracterização florística e estrutural de um fragmento de floresta ombrófila mista em Curitiba, PR, Brasil. Revista Floresta 32:3-16. 
Rondon Neto RM, Watzlawick L, Caldeira M, Schoeninger E. 2002b. Análise florística e estrutural de um fragmento de Floresta Ombrófila Mista Montana, situado em Criúva, RS-Brasil. Ciência Florestal 12:29-37.

Rosário D. 2001. Padrões florísticos e tipos funcionais em floresta com araucária e suas relações com o solo. Dissertação de mestrado, Universidade Federal do Rio Grande do Sul, Porto Alegre.

Sanquetta C, Pizatto W, Péllico-Netto S, Figueiredo Filho A. 2000. Dinâmica da composição florística de um fragmento de Floresta Ombrófila Mista no Centro-Sul do Paraná. Revista Ciências Exatas e Naturais 1:77-88.

Scudeller VV, Martins FR, Shepherd GJ. 2001. Distribution and abundance of arboreal species in the atlantic ombrophilous dense forest in Southeastern Brazil. Plant Ecology 152:185-199.

Seger C, Dlugosz F, Kurasz G, Martinez D, Ronconi E, Melo L, Bittencourt S, Brand M, Carniatto I, Galvão F. 2005. Levantamento florístico e análise fitossociológica de um remanescente de floresta ombrófila mista localizado no município de Pinhais, Paraná-Brasil. Revista Floresta 35:291-302.

Sgrott E. 2003. Fitossociologia da zona ripária no Estado de Santa Catarina. In Anais do I Seminário de Hidrologia Florestal (M. Kobiyama, coord.). Programa de PósGraduação em Engenharia Ambiental - Universidade Federal de Santa Catarina, Alfredo Wagner, p.14-39.

Silva AC, Van Den Berg E, Higuchi P, Oliveira Filho A. 2006. Comparação florística de florestas inundáveis das regiões Sudeste e Sul do Brasil. Revista Brasileira de Botânica 30:257-269.
Silva AC, Berg EV, Higuchi P, Oliveira Filho AT, Marques JJGSM, Appolinário V, Pifano DS, Ogosuko LM, Nunes M. 2009. Florística e estrutura da comunidade arbórea em fragmentos de floresta aluvial em São Sebastião da Bela Vista, Minas Gerais, Brasil. Revista Brasileira de Botânica 32:283-297.

Silva AC, Higuchi P, Aguiar MD, Negrini M, Fert Neto J, Hess AF. 2012. Relações florísticas e fitossociologia de uma Floresta Ombrófila Mista Montana secundária em Lages, Santa Catarina. Ciência Florestal 22: 193-206.

Valério AF, Watzlawick LF, Balbinot R. 2008a. Análise florística e estrutural do componente arbóreo de um fragmento de Floresta Ombrófila Mista em Clevelândia, Sudoeste do Paraná. Revista Acadêmica Ciências Agrárias Ambientais 6:239-248.

Valério AF, Watzlawick LF, Saueressig D, Puton V, Pimentel A. 2008b. Análise da composição florística e da estrutura horizontal de uma Floresta Ombrófila Mista Montana, Município de Irati, PR - Brasil. Revista Acadêmica Ciências Agrárias Ambientais 6: 137-147.

Watzlawick L, Sanqueta C, Valério Á, Silvestre R. 2009. Caracterização da composição florística e estrutura de uma Floresta Ombrófila Mista, no município de General Carneiro (PR). Ambiência 1:229-237.

Webster GL. 1995. The panorama of Neotropical cloud forests. In Biodiversity and conservation of Neotropical Montane Forests (SP Churchill, H Balslev, E Forero, JL Luteyn, eds.). New York Botanical Garden Press, New York, p.53-77. 